Research Paper

\title{
Clinical analysis of EBRT vs TLM in the treatment of early (T1-T2N0) glottic laryngeal cancer
}

\author{
Jing Shen, $\mathrm{Ke} \mathrm{Hu}^{凶}$, Jiabin Ma, Hongnan Zhen, Hui Guan, Wenhui Wang, Fuquan Zhang \\ Chinese Academy of Medical Sciences \& Peking Union Medical College, NO.1 Shuaifuyuan Wangfujing, Dongcheng District, Beijing 100730, People's Republic \\ of China \\ $\square$ Corresponding author: Ke HU: huk@pumch.cn. Chinese Academy of Medical Sciences \& Peking Union Medical College, NO.1 Shuaifuyuan Wangfujing, \\ Dongcheng District, Beijing 100730, People's Republic of China \\ (1) The author(s). This is an open access article distributed under the terms of the Creative Commons Attribution License (https://creativecommons.org/licenses/by/4.0/). \\ See http://ivyspring.com/terms for full terms and conditions.
}

Received: 2020.03.30; Accepted: 2020.09.10; Published: 2020.09.23

\begin{abstract}
Objective: To analyze the clinical efficacy of external beam radiation therapy (EBRT) vs transoral laser microsurgery (TLM) in patients with early glottic laryngeal carcinoma (T1-T2N0) and the effect of treatment choice on vocal function.

Methods: A retrospective analysis of patients with T1-T2N0 glottic laryngeal carcinoma who underwent EBRT or TLM between January 2012 and December 2018 in PUMCH. The Kaplan-Meier method was used to analyze local control, progression-free survival and overall survival, and the VHI-30 scale was used to evaluate the effects of EBRT and TLM on vocal function.

Results: A total of 185 patients, all with pathologically confirmed squamous cell carcinoma, were enrolled. The median age was 62 years (38-88). N0 disease was confirmed by imaging: 142/185 (76.76\%) patients had T1N0 disease, and 43/185 patients (23.24\%) had T2/N0 disease. A total of 91/195 (49.19\%) patients received an EBRT dose of 66-70 Gy/30-35f, at 2.0-2.3 Gy/f. 94/185 (50.81\%) patients received TLM. The median follow-up time was 42 months (12-92), and the 3-year LC, PFS, and OS rates for the EBRT and TLM groups were $96.9 \%$ vs $94.1 \%(p=0.750), 95.3 \%$ vs $93.1 \%(p=0.993)$ and $93.3 \%$ vs $95.4 \%(p=0.467)$, respectively. The VHI-30 scales were used at the baseline showed no significant difference between the two groups $19.20 \pm 3.324$ vs $21.65 \pm 9.80(p=0.250)$, but the EBRT group had a low voice handicap after treatment, $10.24 \pm 6.093$ vs $19.45 \pm 5.112(p=0.001)$ (6 months) and $9.45 \pm 5.112$ vs $14.97 \pm 7.741$ (12 months). No CTCAE grade 3 or above side effects were observed in the EBRT group, but 3 cases of vocal cord stenosis were observed in the TLM group.

Conclusion: The application of EBRT for early glottic laryngeal carcinoma (T1-T2N0) had an obvious curative effect with high LC and OS rates, no serious side effects, and a low voice handicap rate.
\end{abstract}

Key words: glottic laryngeal cancer, external beam radiation therapy, transoral laser microsurgery, voice outcome, VHI

\section{Introduction}

For patients with early glottic laryngeal cancer (T1-T2N0), local treatment, including total/half laryngectomy, transoral laser microsurgery (TLM), and external beam radiation therapy (EBRT), is usually recommended because the probability of lymph node metastasis is $0 \% \sim 2 \%$ [1]. In patients with early glottic laryngeal cancer after total/partial laryngectomy, the 5-year local control (LC) rate can exceed $90 \%$ but with a high voice handicap. To improve the quality of life of patients with early glottic laryngeal cancer, we use other treatments, such as TLM or EBRT, to preserve or maintain the patient's vocal function. Related research has reported that the 5-year LC rate for TLM is approximately 75.81 93.8\% for patients with early glottic laryngeal cancer[2-4]. EBRT is associated with a similar or even better survival outcome, and the 5-year LC rate is approximately $85 \sim 95 \%$ for patients with T1N0 disease $[5,6]$. Although the preferred treatment for early glottic laryngeal cancer is still controversial in terms 
of the therapeutic effect on the tumor, the goals of treatment are to preserve the vocal function of the larynx and aim for the best voice outcome.

In contrast to the effects of TLM on vocal function, the effects of EBRT on the vocal function of patients with early glottic laryngeal cancer are still controversial [7-9]. Therefore, we applied the Voice Handicap Index-30 (VHI-30) scale to evaluate the effect of EBRT and TLM on vocal function. The VHI-30 scale is a self-assessment tool for patients that includes functional, physiological and psychological components. It is widely clinically used. The lower the score is, the better the vocal function will be $[10,11]$.

The aim of this study was to analyze the efficacy of EBRT and TLM in patients with early glottic laryngeal carcinoma (T1-T2N0), the relevant influencing factors, and the use of the VHI-30 scale to evaluate the effect of EBRT and TLM on voice quality.

\section{Methods and materials:}

\section{Patients}

A nonrandomized, longitudinal retrospective analysis was conducted at the Department of Radiation Oncology, Peking Union Medical College Hospital, from January 2012 to December 2018. The inclusion criteria were as follows: patients with T1N0 and limited T2N0 glottic laryngeal cancer; microscopic examination- and biopsy-confirmed pathological squamous cell carcinoma throughout the mouth and throat; an evident parapharyngeal space and prepharyngeal gap on imaging; and N0 disease. Voice outcome and perceptual voice analyses were conducted using the VHI-30, and the data were collected at the baseline of the EBRT or TLM treatment and at various time points during follow-up at 6 months and 12 months.

\section{Treatments}

The patients were separated into two groups for analysis based on treatment strategy. The treatment group consisted of individuals treated with EBRT and the control group consisted of individuals treated with TLM. The details of two treatment strategy were described below.

\section{EBRT}

All patients underwent CT simulation in the supine position (16 rows - Philips Bril-Liance CT Bigbore, Deventer, Netherlands) with head and neck shoulder positioning. The total tumor volume, gross tumor volume (GTV) and CTV (clinical tumor volume) were plotted on the axial CT image. GTV sketching was based on MRI findings. EBRT treatment was administered using a 6-MV X-ray apparatus. For standard fraction (SF) radiotherapy, three-dimensional conformal radiation therapy (3D-CRT) was administered, and for accelerated fraction (AF) radiotherapy, fixed-field intensitymodulated radiation therapy (FF-IMRT), volumetric intensity-modulated radiotherapy (VMAT) or Hi-Art spiral tomography therapy (TOMO) was performed. The CTV included the whole throat area; the GTV included the laryngeal area. The CTV and GTV were evenly placed $5 \mathrm{~mm}$ apart to form the PCTV and PGTV. The doses administered were as follows: PCTV, 60-70 Gy/30-35 f (2 Gy/f) and PGTV, 66-69 $\mathrm{Gy} / 30 \mathrm{f}(2.2-2.3 \mathrm{~Gy} / \mathrm{f})$. The radiation therapy program was generated on an Eclipse or a tomographic treatment planning system with the goal of providing at least $95 \%$ of the prescribed dose to $95 \%$ of the PCTV and PGTV. The organs at risk (OARs) were limited to the spinal cord, at $0.1 \mathrm{cc}<45 \mathrm{~Gy}$. To ensure the accuracy of the treatment, patients receiving TOMO underwent MVCT and online registration every day; patients undergoing FF-IMRT or VMAT received weekly CBCT guidance and were matched online with an error of $3 \mathrm{~mm}$.

\section{TLM}

Transoral CO2 laser microsurgery was carried out under general anesthesia using a Sharpen laser with a digital accolade micromanipulator typically set in continuous or continuous superpulse mode. In most cases, the tumor was first transected to assess the depth of tumor invasion and then resected in two pieces. Resections varied from type I to type $\mathrm{V}$ of the European Laryngological Society (ELS) class action as during the study period, tumors requiring larger resections were treated by RT according to Dutch guidelines. Tumor specimens were pinned on a piece of cork and were sent, along with descriptive drawings, to the pathologist for histological examination. In most cases, standard practice was followed, and additional wound bed biopsies were taken and contained separately. Typically, wound bed biopsies were taken at five different points: four were taken at the edges of the tumor ground, and one deep biopsy was taken in the middle of the tumor ground. If the surgeons found it necessary, extra biopsies could be taken.

\section{Evaluation of vocal function}

The Chinese version of the VHI is a validated 30 -item questionnaire. The VHI-30 is a widely used voice evaluation scale comprising a total of 30 questions that address function, physiology, and emotion. Each part includes 10 questions relating to factors including hoarseness, difficulty breathing, difficulty with pronunciation, and vocal cord distortion. Patients score each item by selecting a 
response based on self-evaluation from a five-point Likert scale ranging from 0 to 4 , as follows: never occurs ( 0 points), almost never occurs (1 point), sometimes occurs ( 2 points), almost always occurs (3 points), always occurs (4 points). The sum of the scores constitutes the total VHI-30 score, which ranges from 0 to 120. A higher score indicates a worse voice-related outcome[10].

\section{Observation indicators and side effects}

The baseline indicators of the patients were collected before radiotherapy, and the patients were followed up every 3 months for 2 years and every 6 months for 2 to 5 years after EBRT. The LC rate was the time interval from the end of treatment to the occurrence of local or regional recurrence. Progression-free survival (PFS) was defined as the time to local or regional recurrence or distant metastasis during or after treatment, and all time intervals were based on the time between the first event and death due to the disease. Overall survival (OS) was defined as the time from diagnosis to death or last follow-up. If no relevant event occurred, PFS or OS was defined from diagnosis to the last follow-up visit.

\section{Statistical analysis}

Statistical analysis was performed using SPSS version 25.0. The chi-square test was used to classify variables. The normality of continuous variables was determined using the Kolmogorov-Smirnov method. Student's t-test was used to evaluate variables with normal distributions, and the Mann-Whitney $U$ test was used for variables with non-normal distributions. Data are expressed as the mean \pm SD. The Kaplan-Meier method was used to estimate the OS, DFS and LC rates, and the significance of prognostic factors for survival was evaluated by a univariate log-rank test. Multivariate analysis of the covariates selected in the univariate analysis was performed using Cox proportional regression. A $p$ value $<0.05$ was considered statistically significant.

\section{Results}

\section{Patient data}

A total of 185 patients were enrolled, as shown in Table 1. All patients had a KPS $\geq 80$ points and a median age of 62 years (38-88 years); $95.14 \%(176 / 185)$ of the patients were male, and $48.65 \%(90 / 185)$ of the patients had a smoking index $\geq 400$. All patients had pathologically confirmed squamous cell carcinoma, and imaging examinations indicated N0 disease. According to the AJCC $7^{\text {th }}$ edition staging criteria, $76.76 \%(142 / 185)$ of the patients had T1N0 disease, of which $65.50 \%(93 / 142)$ had T1a disease, $34.50 \%$
(49/142) had T1b disease, and 23.24\% (43/185) had T2N0 disease. A total of $65.95 \%(122 / 185)$ of the patients had well-differentiated squamous cell carcinomas, $28.65 \% \quad(53 / 185)$ had moderately differentiated cancers, and 5.4\% (10/185) had poorly differentiated cancers. Ninety-one patients $(49.19 \%)$ received EBRT, and ninety-four patients (50.81\%) received TLM treatment. See Table 1 for details.

Table 1. Baseline characteristics

\begin{tabular}{llll}
\hline characteristics & $\begin{array}{l}\text { EBRT group } \\
(\mathrm{n}, \%)\end{array}$ & $\begin{array}{l}\text { TLMgroup } \\
(\mathrm{n}, \%)\end{array}$ & p value \\
\hline $\begin{array}{l}\text { gender } \\
\text { male }\end{array}$ & $84(92.3)$ & $92(97.9)$ & 0.079 \\
female & $7(7.7)$ & $2(2.1)$ & \\
age & & & 0.535 \\
$<65$ & $57(62.64)$ & $63(67.02)$ & \\
$\geq 65$ & $34(37.36)$ & $31(32.98)$ & \\
smoking index & & & \\
$<400$ & $51(56.0)$ & $44(46.8)$ & \\
$\geq 400$ & $40(44.0)$ & $50(53.2)$ & \\
T stage & & & \\
T1 & $78(85.7)$ & $64(68.1)$ & \\
T1a & $52(57.1)$ & $41(43.6)$ & \\
T1b & $26(28.6)$ & $23(24.5)$ & \\
T2 & $13(14.3)$ & $30(31.9)$ & \\
pathology & & & \\
H & $60(65.9)$ & $62(66.0)$ & \\
M & $23(25.3)$ & $30(31.9)$ & \\
L & $8(8.8)$ & $2(2.1)$ & \\
\hline EBRT=external beam radiation therapy; TLM=transoral laser microsurgery; T \\
stage=tumor stage; pathology H=hyper-differentiation, M=middle-differentiation, \\
L=less-differentiation & & & \\
& &
\end{tabular}

\section{Clinical efficacy analysis}

The median follow-up time was 42 months (12-92). For the EBRT group, the 3-year LC rate was $96.9 \%$, and the PFS and OS rates were $95.3 \%$ and $93.3 \%$, respectively. For the TLM group, the 3-year LC rate was $94.1 \%$, and the PFS and OS rates were $93.1 \%$ and $95.4 \%$, respectively. There were no significant differences between the EBRT and TLM groups, as shown in Figure 1.

\section{Subgroup analysis}

For stage T1 ( $\mathrm{n}=142)$, the 3-year LC, PFS and OS rates of the EBRT group $(\mathrm{n}=78)$ and the TLM group $(\mathrm{n}=64)$ were $96.4 \%$ vs $95.6 \%$ ( $\mathrm{p}=0.732), 94.8 \%$ vs $91.1 \%$ $(\mathrm{p}=0.912), 95.6 \%$ and $96.0 \%(\mathrm{p}=0.217)$ respectively. For stage T2 $(n=43)$, the 3-year LC, PFS and OS rates of the EBRT group $(n=13)$ and the TLM group $(n=30)$ were $100 \%$ vs $85.7 \%$ ( $p=0.317), 83.3 \%$ vs $82.9 \%$ ( $p=0.907)$, $75 \%$ and $80.2 \%(p=0.885)$ respectively, as shown in Figure 2.

\section{Side effects}

For the EBRT group ( $n=91)$, a total of 36 out of 91 $(39.6 \%)$ patients had grade 1 acute skin side effects, 54 out of $91(59.3 \%)$ patients had grade 2 acute skin side effects, and 1 out of 91 (1.1\%) patients had grade 3 
acute skin side effects. Patients with grade 1 laryngitis for $22 / 91(24.2 \%)$ patients, and those with grade 2 laryngitis accounted for $69 / 91(75.8 \%)$ patients. In terms of chronic side effects, grade 1 skin reactions occurred in 26/91 (28.57\%) patients, and grade 1 edema occurred in 16/91 (17.58\%) patients. No grade 3 or higher toxic side effects were observed.

For the TLM group ( $\mathrm{n}=94), 1$ patient suffered from asphyxia and hypoxia leading to a vegetative state, 3 patients suffered from massive hemorrhage and underwent reoperation, and 3 patients with chronic side effects underwent surgical treatment for vocal cord stenosis.

\section{Voice outcome}

The VHI-30 scale was used to analyze the vocal function of patients at baseline (before treatment) and at 6 months and 12 months after EBRT and TLM treatment. The results for the EBRT group were $19.20 \pm 3.324,10.24 \pm 6.093$, and $9.45 \pm 5.112$, respectively. For the TLM group, the results were 21.65 \pm 9.80 , $17.54 \pm 8.913$, and $14.97 \pm 7.741$, respectively. There was no significant difference in baseline levels between the two groups, but there was a significant difference in vocal quality between the EBRT group and TLM group at 6 months and 12 months after treatment $(\mathrm{p}<$ 0.01). See Table 3 and Figure 3 for details.

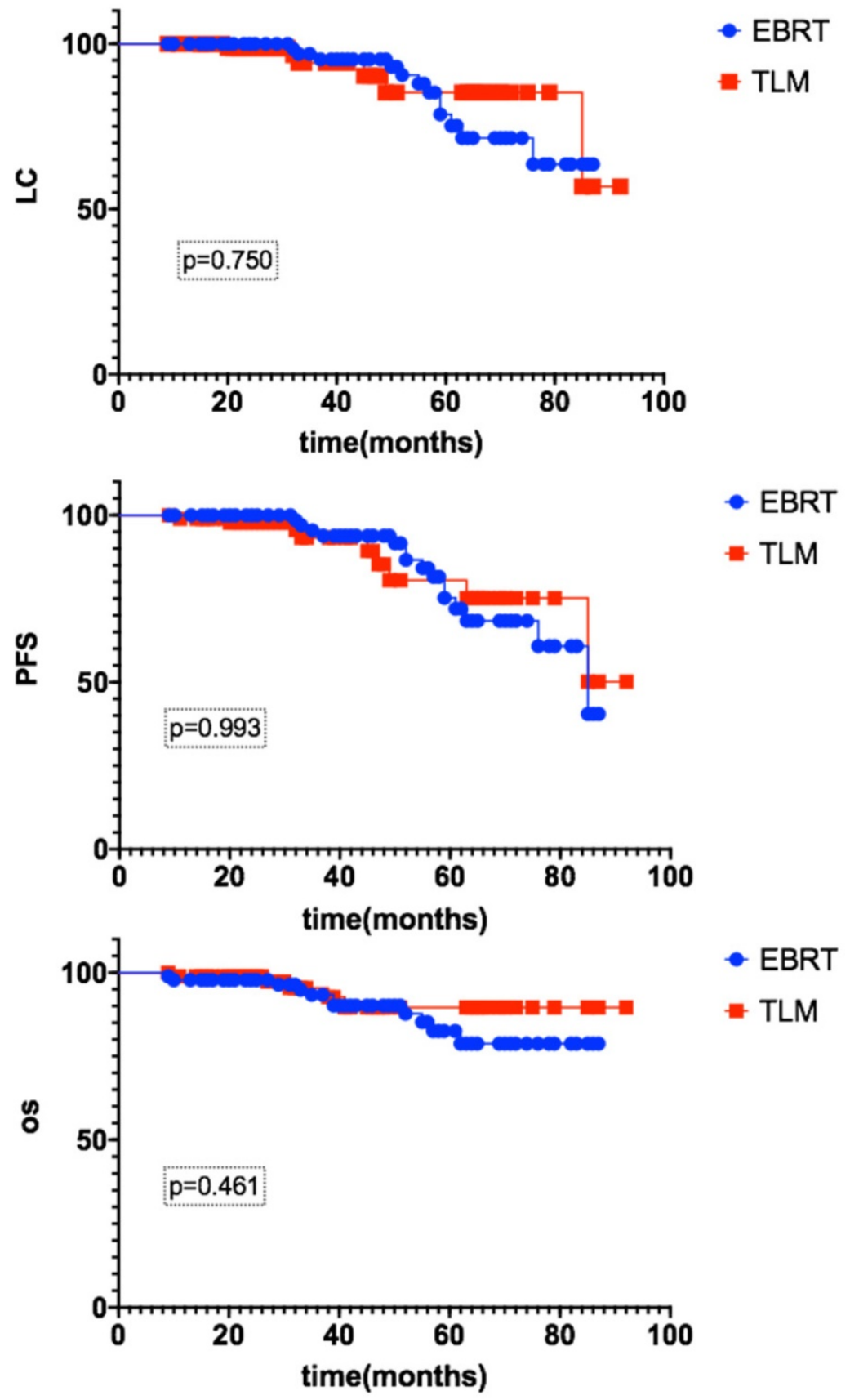

Figure 1. The 3-year LC, PFS and OS rates of early glottic carcinoma patients after EBRT or TLM 

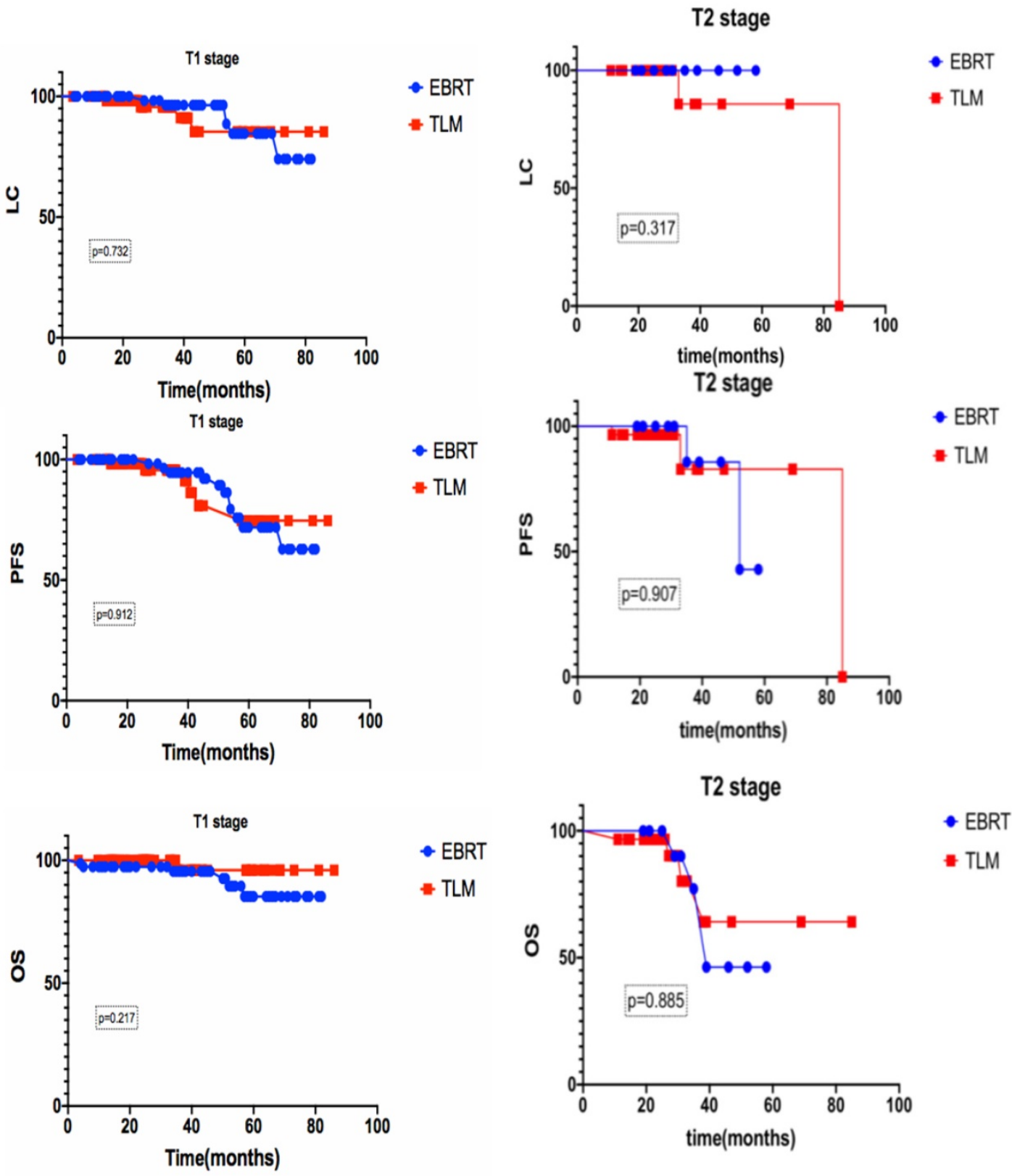

(A) T1 stage

(B) T2 stage

Figure 2. Subgroup analyses of the 3-year LC, PFS and OS rates of early glottic carcinoma patients after EBRT or TLM. Subgroup analyses of the 3-year LC, PFS and OS, (A) stand for T1 stage, (B) stand for T2 stage, there is no significant difference between EBRT and TLM in both stages.

Table 2. Baseline characteristics of the $\mathrm{T} 1$ and $\mathrm{T} 2$ subgroup

\begin{tabular}{|c|c|c|c|c|c|c|c|}
\hline \multirow{3}{*}{$\begin{array}{l}\mathrm{T} 1 \\
\text { subgroup }\end{array}$} & \multicolumn{2}{|l|}{ characteristic } & \multicolumn{2}{|c|}{ EBRT $(n, \%)$} & \multicolumn{2}{|c|}{$\operatorname{TLM}(\mathrm{n}, \%)$} & \multirow{2}{*}{$\begin{array}{l}P \text { value } \\
0.238\end{array}$} \\
\hline & gender & male & 73 & 93.6 & 62 & 96.9 & \\
\hline & & female & 5 & 6.4 & 2 & 3.1 & \\
\hline & \multirow[t]{2}{*}{ age } & $<65$ & 48 & 61.5 & 42 & 65.6 & \multirow[t]{2}{*}{0.470} \\
\hline & & $\geq 65$ & 30 & 38.5 & 22 & 34.4 & \\
\hline & \multirow[t]{2}{*}{ smoking index } & $<400$ & 45 & 57.7 & 34 & 53.1 & \multirow[t]{2}{*}{0.267} \\
\hline & & $\geq 400$ & 33 & 42.3 & 30 & 46.9 & \\
\hline & \multirow[t]{3}{*}{ pathology } & $\mathrm{H}$ & 53 & 67.9 & 42 & 65.6 & \multirow[t]{3}{*}{0.063} \\
\hline & & M & 18 & 23.1 & 21 & 32.8 & \\
\hline & & $\mathrm{L}$ & 7 & 9.0 & 1 & 1.6 & \\
\hline & \multirow[t]{2}{*}{ T stage } & T1a & 52 & 66.7 & 41 & 64.0 & \multirow[t]{2}{*}{0.860} \\
\hline & & $\mathrm{T} 1 \mathrm{~b}$ & 26 & 33.3 & 23 & 36.0 & \\
\hline & anterior joint & no & 40 & 51.3 & 33 & 51.6 & 0.913 \\
\hline
\end{tabular}

\begin{tabular}{|c|c|c|c|c|c|c|c|}
\hline & \multicolumn{2}{|l|}{ characteristic } & \multicolumn{2}{|c|}{ EBRT $(n, \%)$} & \multicolumn{2}{|c|}{$\operatorname{TLM}(\mathrm{n}, \%)$} & \multirow[t]{2}{*}{$P$ value } \\
\hline \multirow{12}{*}{$\begin{array}{l}\mathrm{T} 2 \\
\text { subgroup }\end{array}$} & involved & yes & 38 & 48.7 & 31 & 48.4 & \\
\hline & gender & male & 11 & 84.6 & 30 & 100.0 & 0.082 \\
\hline & & female & 2 & 15.4 & 0 & 0.0 & \\
\hline & age & $<65$ & 9 & 69.2 & 21 & 70.0 & 0.826 \\
\hline & & $\geq 65$ & 4 & 30.8 & 9 & 30.0 & \\
\hline & smoking index & $<400$ & 6 & 46.2 & 10 & 33.3 & 0.621 \\
\hline & & $\geq 400$ & 7 & 53.8 & 20 & 66.7 & \\
\hline & pathology & $\mathrm{H}$ & 7 & 53.8 & 20 & 66.7 & 0.141 \\
\hline & & M & 5 & 38.5 & 9 & 30.0 & \\
\hline & & $\mathrm{L}$ & 1 & 7.7 & 1 & 3.3 & \\
\hline & anterior joint & no & 0 & 0.0 & 4 & 13.3 & 0.198 \\
\hline & involved & yes & 13 & 100.0 & 26 & 86.7 & \\
\hline
\end{tabular}




\section{Failure mode and follow-up treatment}

Local recurrence occurred in 23 patients: 7 patients in stage $\mathrm{T} 1 \mathrm{aN} 0,12$ patients in stage $\mathrm{T} 1 \mathrm{bN} 0$, and 4 patients in stage T2N0. The average time from treatment to recurrence was 17 months (3-45 months), and 18 out of $23(78.26 \%)$ patients experienced recurrence within 2 years after treatment. A total of 18 out of $23(78.26 \%)$ of the recurrence sites were in the vocal cord zone, and 6 out of 23 patients had cervical lymph node metastasis. Five out of 23 patients died: 3 patients had pulmonary metastasis and bone metastasis, and 2 patients died of other diseases. See Table 4 for details.

Table 3. VHI-30 score at baseline and after EBRT or TLM

\begin{tabular}{|c|c|c|c|c|c|c|c|}
\hline & & \multicolumn{3}{|l|}{ EBRT } & \multicolumn{3}{|l|}{ TLM } \\
\hline & & baseline $(\mathrm{n}=91)$ & 6 months after EBRT $(n=90)$ & 12 months after EBRT $(n=84)$ & Baseline $(n=84)$ & 6 months after TLM $(n=80)$ & 12 months after TLM $(n=74)$ \\
\hline \multirow[t]{4}{*}{ Total } & VHI F & $7.78 \pm 2.62$ & $4.24 \pm 3.326$ & $4.02 \pm 2.966$ & $12.71 \pm 6.4$ & $13.95 \pm 6.068$ & $12.23 \pm 5.58$ \\
\hline & VHI P & $7.11 \pm 1.66$ & $3.59 \pm 2.203$ & $3.48 \pm 1.746$ & $5.73 \pm 1.99$ & $2.92 \pm 0.960$ & $2.32 \pm 0.662$ \\
\hline & VHI E & $4.31 \pm 1.082$ & $2.41 \pm 1.931$ & $2.06 \pm 1.819$ & $4.30 \pm 1.079$ & $2.76 \pm 0.845$ & $2.20 \pm 0.741$ \\
\hline & VHI total & $19.20 \pm 3.324$ & $10.24 \pm 6.093$ & $9.45 \pm 5.112$ & $21.65 \pm 9.805$ & $17.54 \pm 8.913^{*}$ & $14.97 \pm 7.741^{*}$ \\
\hline \multirow[t]{4}{*}{$\mathrm{T} 1$ group } & VHI F & $7.58 \pm 2.35$ & $4.19 \pm 2.87$ & $4.25 \pm 4.73$ & $9.16 \pm 5.16$ & $9.77 \pm 6.08$ & $8.22 \pm 5.87$ \\
\hline & VHI P & $7.05 \pm 1.66$ & $3.74 \pm 2.23$ & $3.00 \pm 1.63$ & $4.27 \pm 0.87$ & $3.02 \pm 0.96$ & $2.42 \pm 0.69$ \\
\hline & VHI E & $4.32 \pm 1.06$ & $2.36 \pm 1.87$ & $1.58 \pm 1.55$ & $4.24 \pm 1.07$ & $2.93 \pm 0.83$ & $2.37 \pm 0.76$ \\
\hline & VHI total & $18.95 \pm 3.14$ & $9.92 \pm 7.84$ & $8.83 \pm 6.28$ & $17.94 \pm 7.43$ & $16.05 \pm 7.56^{*}$ & $12.43 \pm 6.82^{*}$ \\
\hline \multirow[t]{4}{*}{$\mathrm{T} 2$ group } & VHI F & $14.00 \pm 3.62$ & $4.54 \pm 5.18$ & $3.99 \pm 2.53$ & $20.88 \pm 7.28$ & $14.71 \pm 7.98$ & $14.13 \pm 6.89$ \\
\hline & VHI P & $4.46 \pm 1.65$ & $2.69 \pm 1.68$ & $3.56 \pm 1.74$ & $4.04 \pm 1.10$ & $3.04 \pm 0.98$ & $2.50 \pm 0.76$ \\
\hline & VHI E & $4.23 \pm 1.19$ & $2.69 \pm 2.16$ & $2.14 \pm 1.84$ & $4.58 \pm 1.15$ & $2.67 \pm 0.80$ & $2.21 \pm 0.71$ \\
\hline & VHI total & $21.69 \pm 3.39$ & $10.30 \pm 5.70$ & $9.55 \pm 4.85$ & $23.60 \pm 14.09$ & $17.93 \pm 11.84^{*}$ & $15.07 \pm 10.15^{*}$ \\
\hline
\end{tabular}

$\mathrm{VHI}=$ voice handicap index, EBRT=external beam radiation therapy, VHI F=voice handicap index function, VHI $\mathrm{P}=$ voice handicap index physical, VHI E=voice handicap index emotion, ${ }^{*}=\mathrm{p}<0.05$

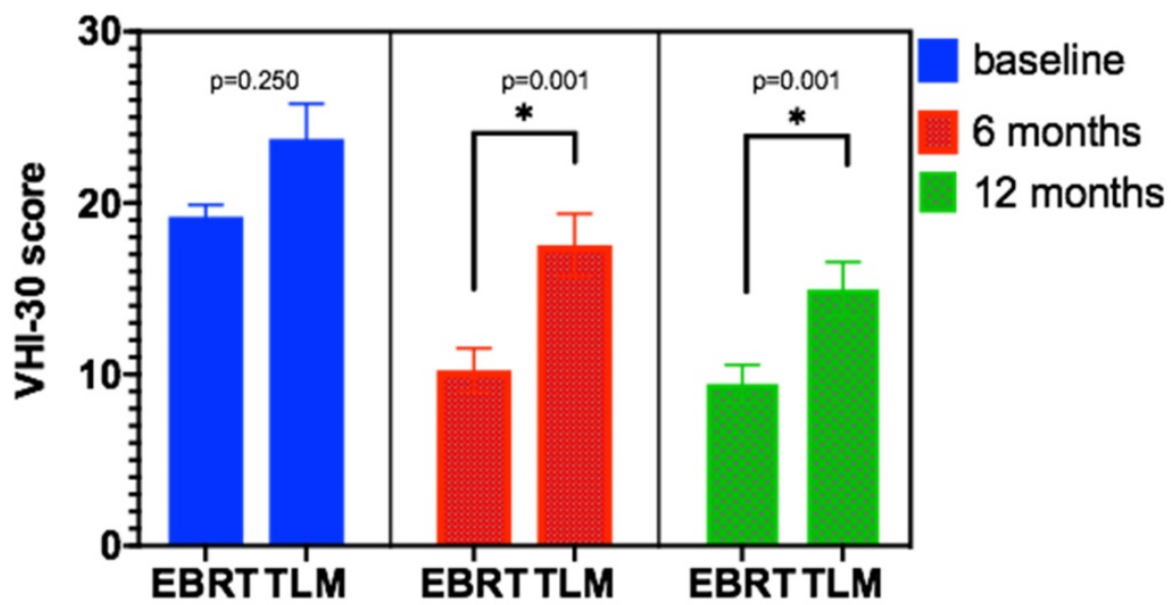

Figure 3. VHI-30 score at baseline and after EBRT or TLM

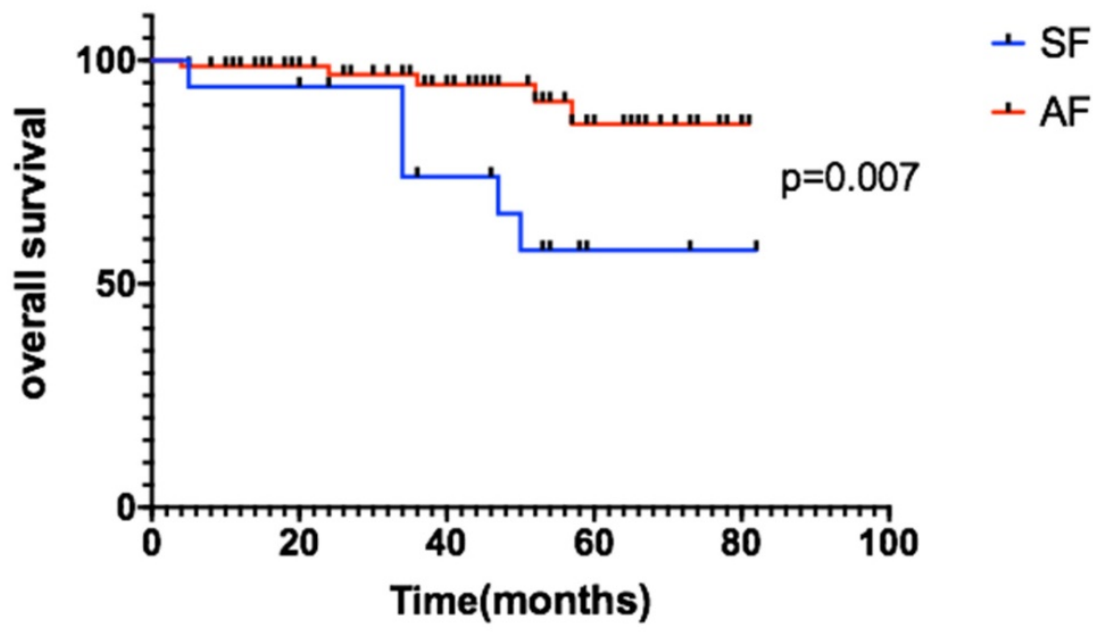

Figure 4. Patients with AF had longer OS than the SF group 
Table 4. Failure pattern for patients treated with EBRT or TLM

\begin{tabular}{lllll}
\hline \multicolumn{5}{c}{ patients(n=185) } \\
\hline & Total $(\mathrm{n}=185)$ & EBRT $(\mathrm{n}=91)$ & $\begin{array}{l}\text { TLM } \\
(\mathrm{n}=94)\end{array}$ & p-value \\
\hline Failure mode & No.(\%) & No. $(\%)$ & No. $(\%)$ & \\
Local & $18(9.73)$ & $12(13.19)$ & $6(6.38)$ & 0.742 \\
Regional lymph node & $6(3.24)$ & $3(3.30)$ & $3(3.20)$ & 0.503 \\
Local and regional lymph & $2(1.10)$ & $1(1.10)$ & $1(1.10)$ & 0.616 \\
node & & & & \\
Distant & $5(2.70)$ & $4(4.40)$ & $1(1.10)$ & 0.425 \\
\hline
\end{tabular}

EBRT $=$ external beam radiation therapy, $\mathrm{TLM}=$ transoral laser microsurgery.

\section{Discussion}

Local treatments are generally applied to preserve the vocal function and improve the quality of life of early glottic laryngeal carcinoma patients. Due to the local infiltration and growth of carcinoma and the advancement of treatment methods, the disease is characterized by a low probability of metastasis. Because EBRT is characterized by a lack of trauma, a good curative effect, and minimal effects on vocal function and quality of life, it is becoming more widely applied for patients with early glottic laryngeal cancer [12, 13]. Because of its relatively non-invasive nature, good curative effect and minimal influence on vocal function and quality of life, TLM has progressively replaced the use of total laryngectomy/hemi-laryngectomy for early glottic laryngeal cancer patients $[4,7]$.

This study demonstrates the use of EBRT and TLM in the treatment of stage T1-T2N0 early glottic laryngeal carcinoma. The total 3-year OS rates of the patients were $93.3 \%$ in the EBRT group and $95.4 \%$ in the TLM group, $p=0.461$. There was no significant difference between the two groups. The 3-year LC rates of the EBRT group and TLM group were 95.5\% vs $89.2 \%, 89.2 \%$ vs $92.9 \%$ and $80 \%$ vs $82.9 \%$, respectively.For subgroup analysis, we compare the baseline characteristics of EBRT and TLM groups, there were no significant difference between two groups (see details in table 2), and also the 3-year LC, PFS and OS of EBRT and TLM in the treatment of stage $\mathrm{T} 1$ or $\mathrm{T} 2$ showed no significant difference (see figure 2 for details). In the stage $\mathrm{T} 1 \mathrm{a}, \mathrm{T} 1 \mathrm{~b}$ and $\mathrm{T} 2$ subgroups, the 3 -year OS rates were $93.6 \%$ vs $93.3 \%$, $90.5 \%$ vs $95.9 \%$, and $60 \%$ vs $64.2 \%$, respectively. PFS in the stage $\mathrm{T} 1 \mathrm{a}, \mathrm{T} 1 \mathrm{~b}$ and $\mathrm{T} 2$ subgroups was $97.4 \%$ vs $89.2 \%, 89.2 \%$ vs $82.5 \%$ and $75 \%$ vs $82.9 \%$, respectively. These results are similar to those reported in other studies [12, 13]. Other studies also compared the efficacy of EBRT to that of TLM [7, 14]. RJ De Santis et al. retrospectively analyzed data for early laryngeal cancer (Tis-T2) at Canada's largest head and neck hospital in 2006-2013 and analyzed 75 patients based on the initial treatment. The patients were divided into two groups: the radiotherapy group and the laser-ablation group. The 5-year DSF rates of the two groups were $90.8 \%$ and $93.3 \%$, respectively, and there were no significant differences in the 5-year LC or PFS rate [2]. A review in Martine 2017 described a comparison of treatments for T2 disease, and 48 studies showed no significant differences between radiotherapy $(\mathrm{n}=3191)$ and TLM $(\mathrm{n}=1156)$ at 5 years $(75.81 \%$ vs $77.26 \%)$ [7]. A phase I clinical study conducted by Leena-Maija Aaltonen et al. compared the efficacy of laser surgery $(n=32)$ to that of EBRT $(n=28)$ for patients with T1a glottic laryngeal carcinoma. The 2-year local recurrence rates were similar: $10 \%$ for the TLM group and $12 \%$ for the EBRT group [8]. Thus, for patients with local early glottic laryngeal carcinoma (Tis-T2N0), EBRT appears to be effective, and the effect of radical radiotherapy is comparable to that of TLM treatment. New radiotherapy techniques, such as inverse intensity-modulated radiotherapy (IMRT), can increase the dose gradient of radiotherapy to reduce the OAR limit, even achieve similar plan quality to Cybernifes system [15]. A number of retrospective studies, including studies by JCOG0701, Tosol Yu, Stokes, Stokes, and Tae Gyu Kim[1, 16-20], have confirmed that compared with the use of SF radiotherapy, the use of AF radiotherapy for large segmentation therapy can increase the LC rate of early glottic laryngeal cancer and provide a survival benefit. The results showed that the application of IMRT combined with the large segmentation method for radiotherapy is reliable [21]. Our study showed that the 3-year OS rates of the SF $(n=17)$ group and the AF $(n=74)$ group were $73.9 \%$ and $94.6 \%$, respectively, $\mathrm{p}=0.007$ (Figure 4). When acute and chronic side effects of the treatment were considered in this study, no grade 3 or higher toxic side effects were found, and there were no statistically significant differences between the radiotherapy technique groups [22]. Most of the data have compared with the results of conventional radiotherapy and laser surgery. Due to the improvement of radiotherapy technology and segmentation mode, we believe that the treatment options of T1 stage patients will be further adjusted.

EBRT is not only effective in treating patients with early glottic laryngeal cancer but can maximize the preservation of the patient's vocal function [11, 23, 24]. In this study, the VHI-30 scale was used to analyze patients before treatment, 6 months after treatment, and 12 months after treatment. VHI-30 is a widely used scale to evaluate voice, including 30 questions and three parts: function, physiology and psychology, studies have analyzed the match between VHI-30 and other multidimensional assessment, showing that the patient's vocal function could be successfully evaluated by VHI-30 [9,11].Compared 
with the TLM group, the voice function of the EBRT group was well preserved. The vocal function of patients before treatment, 6 months after treatment and 12 months after treatment were analyzed by the VHI-30 scale. The vocal function scale scores of the EBRT group and the TLM group were $19.20 \pm 3.324$ vs $21.65 \pm 9.809$ ( $p=0.025), 10.24 \pm 6.093$ vs $17.54 \pm 8.913$ $(\mathrm{p}=0.001)$, and $9.45 \pm 5.112$ vs $14.97 \pm 7.741(\mathrm{p}=0.001)$, respectively. In subgroup analysis, we separate the T1 and T2 stage patients to compare the vocal function after two different treatment, the baseline level of pathological stage of the patients showed no significant difference between two groups, for $\mathrm{T} 1$ stage, the vocal function scale scores at baseline, 6 months and 12 months after treatment of the EBRT group and the TLM group were $18.95 \pm 3.14$ vs $17.94 \pm 7.43 \quad(p=0.27), 9.92 \pm 7.84 \mathrm{vs} 16.05 \pm 7.56$ $(p=0.001)$, and $8.83 \pm 6.28$ vs $12.43 \pm 6.82(p=0.001)$, respectively. For T2 stage, the vocal function scale scores at baseline, 6 months and 12 months after treatment of the EBRT group and the TLM group were $21.69 \pm 3.39$ vs $23.60 \pm 14.09(p=0.37), 10.30 \pm 5.70$ vs $17.93 \pm 11.84(p=0.001)$, and $9.55 \pm 4.85$ vs $15.07 \pm 10.15$ $(p=0.001)$, respectively. There was no significant difference in the baseline level between the EBRT and TLM groups, but there was a significant difference in vocal function between the EBRT group and TLM group at 6 months and 12 months after treatment. The vocal outcome results showed that the patient's vocal function was well preserved after EBRT treatment and that the vocal function at 6 months could predict the functional status within 1 year, which was similar to a previous report $[10,11]$. Relevant studies have also applied these scales to assess the effects of other treatments on vocal function [6]. Compared to surgical treatment or TLM treatment, EBRT retains more complete vocal function $[8,14]$, with significant differences in VHI-F, VHI-P and VHI-E scores [9].

This study has some limitations. First, as a retrospective, single-center study, there may be selection bias. Second, the VHI-30 scale was used to evaluate the impact of voice function. This scale is based on patient self-evaluation rather than a related examination evaluation, which may lead to overestimation of the effects of vocal function. Third, there were more stage T2 stage patients in the TLM group than in the EBRT group, and the effects of TLM may have been underestimated. Despite these limitations, this is a large-scale population study exploring the efficacy of radiotherapy and its effects on voice function in patients with early glottic laryngeal cancer; it has high value as a reference and offers guidance in selecting treatment for patients with early glottic laryngeal cancer.

\section{Conclusion}

EBRT is effective for patients with early glottic laryngeal cancer, offering a high LC rate and OS benefit with tolerable side effects. The use of the VHI-30 scale to evaluate the voice handicap of patients after EBRT for early glottic laryngeal cancer showed high vocal preservation.

\section{Acknowledgements}

\section{Funding}

This work was supported by the Ministry of Science and Technology of the People' s Republic of China (grant number 2016YFC0105207) and National Natural Science Foundation of China (No. U19A2064).

\section{Availability of data and materials}

The datasets used and analyzed during the current study are available from the corresponding author on reasonable request.

\section{Author Contributions}

SJ and HK were responsible for data collection and drafted the manuscript; ZHN, MJB, GH, WWH participated in the design of the study; SJ performed statistic analysis and data interpretation; HK designed the study and revised the manuscript; All authors read and approved the manuscript.

\section{Ethics approval and consent to participate}

The Institutional Review Board (IRB) of Peking Union Medical College Hospital (PUMCH) reviewed the protocol. This is retrospective study. The protocol is rational and scientific. The study accords with principle of ethics.

This is a retrospective study and written human subject consent was unnecessary.

\section{Competing Interests}

The authors have declared that no competing interest exists.

\section{References}

1. Yu T, Wee CW, Choi N, Wu HG, Kang HC, Park JM, et al. Study design and early result of a phase I study of SABR for early-stage glottic cancer. Laryngoscope. 2018; 128: 2560-5.

2. De Santis RJ, Poon I, Lee J, Karam I, Enepekides DJ, Higgins KM. Comparison of survival between radiation therapy and trans-oral laser microsurgery for early glottic cancer patients; a retrospective cohort study. J Otolaryngol Head Neck Surg. 2016; 45: 42.

3. Hendriksma M, Montagne MW, Langeveld TPM, Veselic M, van Benthem PPG, Sjogren EV. Evaluation of surgical margin status in patients with early glottic cancer (Tis-T2) treated with transoral CO2 laser microsurgery, on local control. Eur Arch Otorhinolaryngol. 2018; 275: 2333-40.

4. Carta F, Bandino F, Olla AM, Chuchueva N, Gerosa C, Puxeddu R. Prognostic value of age, subglottic, and anterior commissure involvement for early glottic carcinoma treated with $\mathrm{CO} 2$ laser transoral microsurgery: a retrospective, single-center cohort study of 261 patients. Eur Arch Otorhinolaryngol. 2018; 275: 1199-210.

5. Kitani Y, Kubota A, Furukawa M, Sato K. Prognostic factors for local control in patients receiving radiation therapy for early glottic cancer: anterior 
commissure involvement and effect of chemoradiotherapy. Eur Arch Otorhinolaryngol. 2016; 273: 1011-7.

6. Lee HS, Kim JS, Kim SW, Noh WJ, Kim YJ, Oh D, et al. Voice outcome according to surgical extent of transoral laser microsurgery for T1 glottic carcinoma. Laryngoscope. 2016; 126: 2051-6.

7. Prettyjohns M, Winter S, Kerawala C, Paleri V, the Ncotuatgc. Transoral laser microsurgery versus radiation therapy in the management of T1 and T2 laryngeal glottic carcinoma: which modality is cost-effective within the UK? Clin Otolaryngol. 2017; 42: 404-15.

8. Laoufi S, Mirghani H, Janot F, Hartl DM. Voice quality after treatment of T1a glottic cancer. Laryngoscope. 2014; 124: 1398-401.

9. Kono T, Saito K, Yabe H, Uno K, Ogawa K. Comparative multidimensional assessment of laryngeal function and quality of life after radiotherapy and laser surgery for early glottic cancer. Head Neck. 2016; 38: 1085-90.

10. Fink DS, Sibley H, Kunduk M, Schexnaildre M, Kakade A, Sutton C, et al. Subjective and objective voice outcomes after transoral laser microsurgery for early glottic cancer. Laryngoscope. 2016; 126: 405-7.

11. Hendriksma M, van Loon $Y$, Klop WMC, Hakkesteegt MM, Heijnen BJ, El Hasnaoui I, et al. Quality of life and voice outcome of patients treated with transoral CO2 laser microsurgery for early glottic carcinoma (T1-T2): a 2-year follow-up study. Eur Arch Otorhinolaryngol. 2019; 276: 805-14.

12. Hoebers F, Rios E, Troost E, van den Ende P, Kross K, Lacko M, et al. Definitive radiation therapy for treatment of laryngeal carcinoma: impact of local relapse on outcome and implications for treatment strategies. Strahlenther Onkol. 2013; 189: 834-41.

13. Janssen S, Glanzmann C, Huber G, Studer G. Risk-adapted partial larynx and/or carotid artery sparing modulated radiation therapy of glottic cancer. Radiat Oncol. 2014; 9: 136.

14. Greulich MT, Parker NP, Lee P, Merati AL, Misono S. Voice outcomes following radiation versus laser microsurgery for $\mathrm{T} 1$ glottic carcinoma: systematic review and meta-analysis. Otolaryngol Head Neck Surg. 2015; 152: 811-9.

15. Zhang Y, Chiu T, Dubas J, Tian Z, Lee P, Gu X, et al. Benchmarking techniques for stereotactic body radiotherapy for early-stage glottic laryngeal cancer: LINAC-based non-coplanar VMAT vs. Cyberknife planning. Radiat Oncol. 2019; 14: 193.

16. Kodaira T, Kagami Y, Shibata T, Shikama N, Nishimura Y, Ishikura S, et al. Results of a multi-institutional, randomized, non-inferiority, phase III trial of accelerated fractionation versus standard fractionation in radiation therapy for T1-2N0M0 glottic cancer: Japan Clinical Oncology Group Study (JCOG0701). Ann Oncol. 2018; 29: 992-7.

17. Rao SN, Zafereo M, Dadu R, Busaidy NL, Hess K, Cote GJ, et al. Patterns of Treatment Failure in Anaplastic Thyroid Carcinoma. Thyroid. 2017; 27: 672-81.

18. Okubo M, Nishimura Y, Shibata T, Nakamatsu K, Kanamori S, Tachibana I, et al. Definitive radiation therapy for moderately advanced laryngeal cancer: effects of accelerated hyperfractionation. Jpn J Clin Oncol. 2010; 40: 944-8.

19. Kim TG, Ahn YC, Nam HR, Chung MK, Jeong HS, Son YI, et al. Definitive radiation therapy for early glottic cancer: experience of two fractionation schedules. Clin Exp Otorhinolaryngol. 2012; 5: 94-100.

20. Zumsteg ZS, Riaz N, Jaffery S, Hu M, Gelblum D, Zhou Y, et al. Carotid sparing intensity-modulated radiation therapy achieves comparable locoregional control to conventional radiotherapy in T1-2N0 laryngeal carcinoma. Oral Oncol. 2015; 51: 716-23.

21. Sher DJ, Timmerman RD, Nedzi L, Ding C, Pham NL, Zhao B, et al. Phase 1 Fractional Dose-Escalation Study of Equipotent Stereotactic Radiation Therapy Regimens for Early-Stage Glottic Larynx Cancer. Int J Radiat Oncol Biol Phys. 2019; 105: 110-8.

22. Huang $G$, Luo $M$, Zhang J, Liu $H$. The voice quality after laser surgery versus radiotherapy of T1a glottic carcinoma: systematic review and meta-analysis. Onco Targets Ther. 2017; 10: 2403-10.

23. Aaltonen LM, Rautiainen N, Sellman J, Saarilahti K, Makitie A, Rihkanen H, et al. Voice quality after treatment of early vocal cord cancer: a randomized trial comparing laser surgery with radiation therapy. Int J Radiat Oncol Biol Phys. 2014; 90: 255-60.

24. Angadi V, Dressler E, Stemple J. A Multidimensional Study of Vocal Function Following Radiation Therapy for Laryngeal Cancers. Ann Otol Rhinol Laryngol. 2017; 126: 483-92. 